Meta

Journal des tradlucteurs

Translators' Journal

\title{
Le travail perlocutoire de la traduction Macbeth québécois
}

\section{Annie Brisset}

Volume 34, numéro 2, juin 1989

URI : https://id.erudit.org/iderudit/002149ar

DOI : https://doi.org/10.7202/002149ar

Aller au sommaire du numéro

Éditeur(s)

Les Presses de l'Université de Montréal

ISSN

0026-0452 (imprimé)

1492-1421 (numérique)

Découvrir la revue

Citer cet article

Brisset, A. (1989). Le travail perlocutoire de la traduction Macbeth québécois. Meta, 34(2), 179-194. https://doi.org/10.7202/002149ar d'utilisation que vous pouvez consulter en ligne.

https://apropos.erudit.org/fr/usagers/politique-dutilisation/ 


\title{
LE TRAVAIL PERLOCUTOIRE DE LA TRADUCTION MACBETH QUÉBÉCOIS
}

ANNIE BRISSET

Université du Québec à Montréal, Canada

Sur la lande où dansent les sorcières, un tambour annonce l'arrivée de Macbeth. C'est pour le spectateur la première entrée en scène du personnage principal de la tragédie:

\author{
«Drum within» \\ 3 WITCH \\ A drum, a drum! \\ Macbeth doth come. (1962: 847)
}

Michel Garneau, l'auteur de la version québécoise, choisit d'annoncer Macbeth au son d'une autre musique :

TROISIÈME

«Violon»

Le vialon, le vialon, Macbeth s'en vient 'citte ! (1977:19)

Par métonymie, le violon désigne au Québec le violonneux et le rythme sautillant de la gigue. S'il passe presque inaperçu à la lecture, ce marqueur est doté d'un formidable pouvoir d'actualisation, car pour l'auditoire, il transforme instantanément l'Écosse fictive et lointaine de Macbeth en un lieu connu et familier. De plus, l'insertion de cette musique populaire typiquement québécoise fonctionne, au second degré, comme un puissant opérateur discursif à partir du moment où elle conduit le spectateur à saisir que le lieu dont on parle (Écosse / fiction) se superpose au lieu d' où l'on parle (Québec / réalité). Par la fusion même de son object et de son lieu d'énonciation le dialogue de la tragédie shakespearienne se transforme en un discours à double entente qui agira sur la conscience de l'auditoire. Suivant la théorie des actes de langage, nous appellerons cela le travail perlocutoire de la traduction'. Nous voulons désigner ainsi l'ensemble des transformations qui confèrent au texte d'arrivée une fonction persuasive, absente du texte de départ.

D'ordinaire, une actualisation théâtrale consiste à homologuer l'espace et le temps de l'original à une réalité familière au spectateur. Ainsi Robert Lalonde redéploie-t-il l'action des Trois Sours de Tchékhov dans le Québec des années cinquante, modifiant le nom et jusqu'à la fonction des personnages ${ }^{2}$. Loin d'actualiser globalement le texte original, Michel Garneau préserve au contraire l'identité intrinsèque de Macbeth, mais il introduit ponctuellement des modifications qui transportent la tragédie de Shakespeare sur une autre scène, dans un passé recomposé où elle rebondit sous la forme d'une allégorie de la tragédie réelle, vécue ici autrefois, éclairant alors pour le spectateur la situation vécue ici et maintenant. Ainsi la traduction intègre-t-elle doublement le propos shakespearien au discours social ${ }^{3}$ qui rend compte de la réalité québécoise.

La première intégration se joue, comme on l'a vu, dans la spatialité: l'ailleurs étranger, fictif et lointain de Macbeth s'actualise au Québec par le choix explicite de la langue d'arrivée que souligne, sur la couverture de l'ouvrage, la mention «traduit en qué- 
bécois». La seconde intégration opère dans la temporalité: sous l'effet de certains marqueurs tels que la forme archaïque de la langue de traduction, le récepteur québécois perçoit l'éloignement temporel du lieu que d'autres marqueurs lui rendent familier. Contradictoires en apparence, ces deux déplacements médiatisés par la traduction réalisent une actualisation historique de la fiction shakespearienne, qui rend la fusion du dialogue fictif et du discours social ambiant beaucoup plus subtile qu'une transposition affichée des lieux et des personnages. C'est néanmoins dans cette interaction du propos, de l'espace et du temps que s'exerce le travail perlocutoire de la traduction.

La notion de chronotope ${ }^{4}$, qui désigne la conjonction donnée d'un espace et d'un temps, nous permettra d'illustrer la manière dont les marques d'actualisation font tourner les courroies du discours, c'est-à-dire comment elles permettent au spectateur d'enclencher sur le dialogue de Macbeth les valeurs et les idées qui, dans le discours social, rendent compte du fait québécois, ou de ce qu'on appelle encore la «québécité».

Dans sa version québécoise, la représentation de Macbeth fait intervenir trois principaux espaces-temps, à savoir : 1) le chronotope de l'original : Écosse / temps mythique; 2) le chronotope du spectateur: Québec / temps de la réception. À chacun de ces chronotopes correspond une configuration discursive, autrement dit: qui parle, de quoi et comment? Dans le premier se déploie le dialogue imaginé par Shakespeare. Dans le deuxième se déploie le même dialogue refaçonné par la traduction. Dans le troisième enfin s'exerce de façon infiniment plus vaste et plus complexe le discours de la société québécoise dans lequel s'inscrivent, bien sûr, le traducteur comme le spectateur.

A partir de ces données nous pouvons schématiser l'interaction des trois configurations discursives autour de leurs chronotopes respectifs.

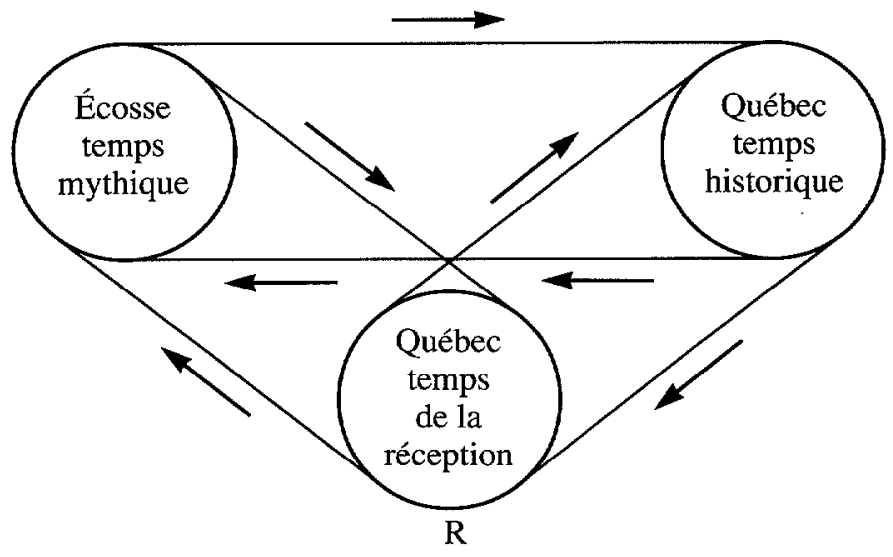

Ce schéma, à la périphérie duquel est situé le récepteur (R), montre qu'il existe pour ce dernier une multiplicité de parcours sémantiques au fur et à mesure du déroulement de la pièce. Loin d'être libres et aléatoires, ces parcours sont aiguillés par des éléments divers, notamment par les actualisateurs qui marquent l'espace et le temps et qui induisent le récepteur à projeter le contenu du dialogue shakespearien sur l'un ou l'autre des chronotopes québécois (historique ou contemporain).

La pièce comporte, bien sûr, des séquences plus compatibles que d'autres avec la problématique québécoise et il faut s'attendre que la traduction exploite ces passages les plus propices à une sémantisation axio-idéologique en fonction des données du Québec. On comprend aussi que, ponctuellement, certains éléments du texte original facilitent ou entravent l'amalgame de ce dernier avec le discours de la société québécoise. Le traduc- 
teur pourra donc être porté à souligner ou à supprimer ces éléments selon qu'ils renforcent ou détruisent le potentiel d'investissement axio-idéologique que la fiction étrangère offre au discours social qui la reçoit. Même absents du texte d'arrivée, ces marqueurs y exercent une fonction signalétique puisque leur silence libère, d'un chronotope à un autre, le passage du discours porté par le dialogue théâtral. À l'inverse, le maintien de ces éléments peut barrer la route à des idéologèmes ${ }^{5}$ du discours social et contenir la saisie du texte-source dans son chronotope initial (Écosse/temps mythique). Telle sera du moins la perception du spectateur à la merci de la traduction dont le travail est ici comparable à celui d'un aiguillage.

Cet ajustement du texte original, qui intervient à des degrés divers sur n'importe quel texte mais dont le traducteur souvent n'a pas même conscience, fait apparaitre les codes régissant le discours de la société où viennent s'insérer les textes étrangers filtrés par elle. C'est en cela qu'il intéresse la théorie de la traduction. Le circonscrire permet de mieux connaître les déterminations institutionnelles de l'opération traduisante ainsi que le rôle, mal connu et bien peu étudié, du sujet de cette opération, c'est-à-dire le traducteur lui-même, relais des normes du discours social et de l'institution qui les instaure et les sanctionne ${ }^{6}$.

À partir d'un seul indice (la substitution d'un instrument de musique), nous venons de formuler quelques principes dont nous allons vérifier le fonctionnement à l'échelle du texte. Commençons par identifier les transformations spatio-temporelles en comparant la désignation des lieux dans les indications de scène du texte original et de la traduction québécoise.

Désignation des lieux dans les indications de scène

Scotland; England

I.1 A desert Heath

I.2 A Camp near Forres

I.3 A Heath

I.4 Forres. A Room in the Palace

I,5 Inverness. Macbeths Castle

I.6 Before the Castle

I.7 A Room in the Castle

II.1 Inverness. Court within the Castle

II,4 Without the Castle

III,1 Forres. A Room in the Palace

III, 2 Another Room in the Palace

III,3 A Park, with a Road leading to the Palace

III,4 A Room of State in the Palace

III,5 A Heath

III,6 Forres. A Room in the Palace

IV,1 A Cavern

IV,2 Fife, Macduffs Castle

IV,3 England. Before the Kings Palace
En Écosse, en Angleterre

Un brûlé

- O -

Le brûlé

Devant le château de Macbeth

Au loin dans la plaine

Au château de Macbeth

La grande salle

Une chambre

Cour dans le château de Macbeth

(Inverness)

- O -

$-\mathrm{O}$ -

- O -

Au bord de la forêt

Salle à manger

Le brûlé

A Forres

- O -

Le château de Macduff

En Angleterre 
$\mathrm{V}, 1$ Dunsinane. A Room in the Castle

$V, 2$ The Country near Dunsinane

$\mathrm{V}, 3$ Dunsinane. A Room in the Castle

V,4 Country near Birnam Wood

V,5 Dunsinane. Within the Castle

V,6 A Plain before the Castle

V,7 Another Part of the Plain
Chez Macbeth

Dans la campagne

Chez Macbeth

Près de la forêt

Chez Macbeth

Devant le château

Ailleurs dans la plaine

Concordance des noms de lieux

\section{SHAKESPEARE}

England
Scotlant
Forres
Dunsinane
Inverness
Birnam
Fife
NOMS PROPRES
castle
palace
room
room of state
court
park
heath
country
plain
wood
road
camp
cavern

NOMS COMMUNS

TOTAL

\section{GARNEAU}

$\begin{array}{ll}\text { Angleterre } & 2 \\ \text { Écosse } & 1 \\ \text { Forres } & 1 \\ & 0 \\ \text { Inverness } & 1 \\ & 0 \\ & 0\end{array}$

\section{6}

10

château

5

chambre

salle à manger

la grande salle

cour

brûlé

campagne

plaine

forêt

Ces tableaux comparatifs découvrent la simplification réductrice de la version québécoise où subsiste à peine la moitié des lieux initialement présentés. De plus, ceux-ci reçoivent une désignation plus vague puisque les personnages évoluent «au bord de la forêt» (A Park with a Road Leading to the Palace), «dans la campagne» (The Country near Dunsinane) ou «chez Macbeth» (Dunsinane. A Room in the Castle).

Cette neutralité du lieu de l'action apprivoise le mythe inquiétant. Ainsi disparaît la caverne tandis que l'espace désert de la lande revient sous la forme domestiquée d'un «brûlé». Le lecteur ne peut manquer d'associer cet équivalent québécois du brûlis au sou- 
venir de la colonie et de ses pionniers défricheurs, une colonie jalonnée d'ouvrages défensifs, de forts et de châteaux, mais non de palais. Dans les indications de scène Garneau n'a reproduit aucune des nombreuses occurrences du mot «palaze». Il a néanmoins traduit «castle», qui représente un degré moins élevé dans la hiérarchie des habitations, non sans réduire l'importance de ce terme qu'il n'a rendu qu'une fois sur deux. Rien d'étonnant qu'il ait alors transformé «A Room of State» en «salle à manger», dans un registre plus commun que celui auquel appartient une salle d'apparat.

Pour expliquer ce décalage systématique vers le bas, on peut invoquer le caractère dialectal de la langue-cible, qui limiterait les possibilités offertes au traducteur. Mais en toute logique, l'entreprise qui consiste à traduire une œuvre canonique «en québécois» vise au contraire à dédialectiser cette langue et à prouver que c'est une langue à part entière. Et l'on sait que la propriété des langues est de pouvoir exprimer n'importe quelle réalité en créant au besoin les moyens qui leur font défaut. Selon le code graphique et phonétique qu'il a choisi, Garneau aurait pu traduire «the Kings Palace» par «el' pala's $d u$ roé». Sa constante omission du palais signale qu'il existe un conflit entre ce que la langue peut dire et ce que le traducteur choisit d'exprimer. La limitation est à chercher ailleurs que dans le registre d'expression ou la nature dialectale de la langue et bien plutôt dans la mise en rapport du contenu de la pièce et du fait québécois, que cette langue est certainement chargée d'exprimer et de valoriser. Par opposition au palais, le château est aussi bien une demeure seigneuriale qu'un ouvrage défensif, une enceinte protectrice entourée de remparts, trouée de meurtrières, hérissée de créneaux et de tours de guet. En tant que fortification et résidence seigneuriale, le château est une réalité qui appartient à l'histoire du Québec, contrairement au palais. D'où la résonance que prend, par cette mémoire historique, l'attaque du château de Macbeth :

\section{MACBETH}

Fa's accrocher toutes nos bannières après 'es créneaux !

Ca m'fa't rien d'entende crier qu'y s'en viennent, l'château

Est assez fort pour prende toutes leus attaques; on va 'es laisser

S'évartuer comme des bons jusqu'à temps qu'y creuvent ed'faim

Pis qu'les fieuves 'es mangent tout crus. R'marqu', si-z-éta'ent pas

Renforcis en nombre par eune bande de traïtes qui s'r'aient s'posès

D'ête d'not'bord, on s'ra't sorti'es défier face à face

Dans l'corps à corps pour les r'culer jusque cheuz-eux ! (140)

On verrait bien ce même passage dans une reconstitution historique québécoise rapportant l'attaque d'un fort par les Indiens ou les Anglais, événement somme toute banal comme on en trouve dans les livres d'histoire qui ont nourri l'imagination du public. La relation est d'autant plus facile à établir que Michel Garneau a estompé les noms propres. Dans les tableaux qui précèdent, on relève que les deux tiers des toponymes fixant le cadre de l'action dramatique sont effacés. Le chiffre est considérable, et cette observation qui vaut pour les indications de scène est confirmée par le texte lui-même. Témoin parmi d'autres, la prophétie autour de laquelle se dénoue la tragédie :

Macbeth shall never venqui s's be until

Great Birnam wood to high Dunsinane hill

Shall come against him. (861. Nous soulignons)

Macbeth s'ra pas vaincu tant qu'la forêt descendra pas d'la colline

(106. Nous soulignons)

Non seulement Garneau supprime le nom des lieux, mais il supprime également leurs attributs: "great Birnam wood», «high Dunsinane hill» sont des lieux surdimen- 
sionnés, extraordinaires, entournés d'une aura tout aussi mythique que la forêt de Brocéliande. Ramenés par le traducteur à des proportions normales, ils acquièrent la banalité d'un environnement rural quotidien. S'estompe ainsi l'écho des chroniques de Holinshed où Shakespeare a puisé la trame de son histoire. Macbeth déraciné de la mythologie anglo-saxonne est transplanté sur un sol qui ressemble à celui de la NouvelleFrance. L'espace-temps de la tragédie shakespearienne se déplace, ne serait-ce que par intermittence, vers un Québec historiquement localisable.

On voit ici comment la fiction du texte original devient embrayable sur la réalité québécoise moyennant la suppression ou l'adjonction de certains marqueurs. Garneau élimine d'ailleurs ouvertement certains passages qu'il juge dénués de pertinence. Voici par exemple comment il explique l'élimination de toute une séquence au milieu de la troisième scène du quatrième acte: «J'traduis pas du vers 139 au vers 161 (Édition Oxford University Press) parc'c'est la scène avec le docteur où l'on parle du don de guérisseur du roi d'Angleterre, une scène de ventilation comme on dit au cinéma, qui n'a rien à faire avec l'action dramatique pis qui sonne même pas très Shakespeare» (1977: 121). I1 prétend ainsi restituer à la pièce sa pureté originelle, que présumément ce passage adultère. La tragédie de Macbeth, on le sait, est reconnue pour être «l'un des textes les plus sujets à caution de tout le canon shakespearien» (Legris 1977:13), mais les interpolations en ont été depuis longtemps repérées par la critique. Celle que Garneau élimine n'est pas des plus certaines ${ }^{7}$. Par ailleurs, cette séquence ne comporte aucune équivoque et ne suscite aucune inconséquence verbale par rapport à ce qui suit ou précède. Et s'il existe dans la scène un passage que la critique a mis en cause sur le plan de la pertinence dramatique, c'est moins la brève tirade du médecin que le dialogue méandreux qu'elle interrompt. Le traducteur n'a pourtant rien sacrifié de ce dialogue. Il en a bien au contraire souligné l'intensité dramatique. La véritable motivation est donc ailleurs, si l'on admet en principe qu'une suppression de cette envergure, soulignée de surcroît par le traducteur, relève d'une logique discursive. Rétablissons le passage supprimé :

\section{Enter a Doctor}

\section{MALCOLM}

Well; more anon. Comes the king forth, I pray you?

\section{DOCTOR}

Ay, sir; there are a crew of wretched spuls

That stay his cure; their malady convinces

The great essay of art; but at his touch,

Such sanctity hath heaven given his hand,

They are presently amend.

MALCOLM

I thank you, doctor.

\section{Exit Doctor}

MACDUFF

Whats the disease he means?

\section{MALCOLM}

Tis call'd the evil :

A most miraculous work in this good king,

Which often, since my here-remain in England,

I have seen him do. How he solicits heaven,

Himself best knows; but strangely-visited people,

All swoln and ulcerpus, pitiful to the eye, 
The mere despair of surgery, he cures ;

Hanging a golden siamp about their necks,

Put on with holy prayers; and 'tis spoken

To the succeeding royalty he leaves

The healing benediction. With this strange virtue,

He hath a heavenly gift of prophecy.

And sundry blessings hang about his throne

That speak him full of grace.

MACDUFF

See, who comes here?

MALCOLM

My countryman; but yet I know him not. (864)

Pourquoi le traducteur a-t-il jugé nécessaire, impératif même, de supprimer cette séquence dont il prend soin de souligner le caractère accessoire en disant qu'elle est une «scène de ventilation»? Prenons-le au mot. Si ce passage est rejeté pour sa fonction d'intermède, c'est en raison même de sa valeur disruptive. En quoi était-il donc important de ne pas interrompre la scène où ce passage est intercalé ? Pour le comprendre, récapitulons brièvement le propos de la scène.

Réfugié en Angleterre après l'assassinat du roi Duncan, son père, Malcolm, héritier légitime du trône d'Écosse, apprend de Macduff, général déserteur venu le rejoindre en exil, l'état d'asservissement dans lequel est plongée l'Écosse dominée par l'usurpateur Macbeth. Les deux hommes se jaugent puis dressent le bilan des forces qui leur permettront d'organiser le soulèvement avec l'appui du roi d'Angleterre. Leur entretien est interrompu par le médecin qui vante les dons surnaturels du monarque dont la venue est imminente. Sur les entrefaites arrive le général Ross, apportant avec lui des nouvelles accablantes sur les derniers forfaits du tyran d'Écosse, en particulier le massacre de la famille de Macduff. Celui-ci d'abord terrassé par le malheur qui le frappe, se ressaisit et s'engage à prendre la tête de la rébellion qui libérera l'Écosse opprimée.

Construites en parallèle, les deux séquences font passer de la tragédie nationale, vécue par tout un peuple, à la tragédie individuelle, qui galvanise la victime au point de mobiliser toutes les forces de la résistance. À la charnière des deux séquences qui forment la scène, intervient le panégyrique du roi d'Angleterre: un intermède qui, décidément, n'a aucun rapport avec l'action dramatique et moins encore avec la situation du Québec. Car il faut ignorer l'histoire de la Nouvelle-France pour ne pas reconnaître dans le dialogue une frappante analogie avec la conquête et le soulèvement des patriotes, ni même y entendre tout simplement l'écho des aspirations souverainistes du moment. Voyons comment, sur le contenu discursif de la pièce, le traducteur enclenche certaines données propres au discours de la société dont il fait partie.

Le mot "Scotland», marqueur de l'action dramatique, revient de nombreuses fois dans la scène et constitue l'objet central du dialogue. Il est employé en alternance avec des noms communs tels que «nation», «state», «country», qui s'accompagnent de qualificatifs négativement chargés tels que "poor», «miserable», "down-fall'n». Nous avons regroupé sous forme de tableau les occurrences de tous ces noms qui désignent le lieu de l'action dramatique et nous les avons accompagnés de leurs déterminants (articles, adjectifs possessifs, démonstratifs et qualificatifs). En regard nous avons placé le paradigme équivalent extrait de la traduction. Dans un autre tableau nous avons établi la concordance entre le texte de départ et le texte d'arrivée; les chiffres indiquent le nombre d'occurrences de chaque terme. 
Désignations de l'Écosse (IV, 3)

\section{SHAKESPEARE}

-0 -

our down-faill' $n$ birthdom

Scotland

- 0 -

poor country

- 0 -

our country

-0 -

-0 -

my poor country

poor state

Scotland

Scotland

Scotland

nation miserable

Scotland

thine and my poor country

Scotland

poor country

our mother

our grave

-0 -

-0 -

-0 -

Scotland

Scotland
GARNEAU

cheuz-nous

not'droit d'exister ram'né à rien not'pau'pays

not'pays

pauv'pays

mon pauv'pays

not pauv'pays

c't'un pays

c't'un pays

mon pauv'pays

l'pays

Écosse

pauv'pays

pauv'Écosse

nation ben misérabe

mon pays

l'pauv'pays / l'tien pis l'mien

not'Écosse

not'pauv'pays

not'mére-patrie

tombe

fosse commune

cheuz-nous

cheuz-nous

Écosse

- 0 - 
Concordance des désignations de l'Écosse (IV, 3)

\section{SHAKESPEARE}

Scotland

birthdom

country

state

nation

mother

grave

\section{GARNEAU}

8 Écosse

1 droit d'exister 1

5 pays 13

1 (pays) 1

1 nation 1

1 mère patrie 1

1 fosse commune 1

\begin{tabular}{llll}
\hline TOTAL & 10 & TOTAL & 21
\end{tabular}

\begin{tabular}{llll}
$\begin{array}{l}\text { dowm-fall'n } \\
\text { poor } \\
\text { miserable }\end{array}$ & 1 & $\begin{array}{l}\text { ram'né à rien } \\
\text { pauv' } \\
\text { ben misérabe }\end{array}$ & $\begin{array}{l}1 \\
9 \\
1\end{array}$ \\
\hline TOTAL & 1 & TOTAL & 11
\end{tabular}

\begin{tabular}{llll} 
our & 4 & not' & 7 \\
thine and mine & 1 & l'tien pis I'mien & 1 \\
my & 1 & mon & 3 \\
\hline
\end{tabular}

$\begin{array}{llll}\text { TOTAL } & 6 & \text { TOTAL } & 11\end{array}$

$\begin{array}{lll}\text { TOTAL GÉNERAL } & 31 \quad \text { TOTAL GÉNÉRAL } & 47\end{array}$

Dans le texte original, la fréquence du toponyme «Scotland» prédomine sur celle de tous les noms communs qui, dans le contexte précis de la pièce, désignent la même entité spatiale. La traduction de Garneau estompe au contraire ce toponyme dont elle réduit la fréquence de moitié au profit des éléments qui forment le syntagme "not' pauv' pays». La traduction en inverse la proportion par rapport à celle de «Scotland». Avec ses variantes «pau(v)' pays» et «mon pauv' pays, ce syntagme porté à 7 occurrences contre 4 du syntagme original équivalent, acquiert à la lecture et à l'écoute une prégnance particulièrement forte.

La comparaison des deux listes révèle par ailleurs d'intéressantes modifications. Le paradigme original des noms communs est hiérarchisé : il s'ouvre sur «birthdom" et se termine par «grave». Chez Garneau, la même liste est encadrée par "cheuz-nous», expression ajoutée sans que le texte original ne le justifie par la présence d'une notion équivalente:

Let us seek out some desolate shade, and there

Weep our sad bosoms empty. (863)

Quand j'pense à cheuz-nous, j'ai jusse envie d'en trouver un coin

Tranquille à l'ombre pour m'laisser fère pis brailler tout mon soûl (116) 
Cette première apparition de «cheuz-nous» est loin d'être anodine sur le plan de la stratégie discursive, car les deux vers cités ouvrent la scène. L'interpolation de «quand j'pense à cheuz-nous» est donc littéralement une mise en exergue qui d'emblée mobilise l'attention du spectateur et oriente l'interprétation du dialogue qui va suivre en fonction de la réalité québécoise. Il ne faut pas perdre de vue, et la graphie est là pour l'attester, que la nature phonétique de cette proposition joue au Québec le rôle d'un actualisateur. Celui-ci est d'autant plus sensible à l'auditoire que l'usage du franco-québécois pour représenter Shakespeare est inédit. Il remplit donc avec beaucoup d'efficacité auprès du spectateur ce que Jakobson appelait la fonction phatique, celle qui garantit que la communication passe bien. La conséquence de cet actualisateur spatial et discursif est que, dans la logique du discours ambiant, le spectateur québécois peut prendre à son compte l'expression «quand j'pense à cheuz-nous» avec sa suite prédicative «j'ai jusse envie d' [...] brailler tout mon soûl». Dans la foulée de ces deux vers introductifs la traduction effectue un glissement qui ne peut qu'induire un peu plus la projection de la réalité québécoise sur le contenu de la scène originale :

Let us rather $[. .$.

Bestride our down-fall' $n$ birthdom ; (863. Nous soulignons)

Moé, j'pense qu'on f're't mieux d'[...] défende

Not'droét d'exister qui s'strouve ram'né quasiment à rien;

(116. Nous soulignons)

Pour le spectateur, le rapprochement avec le Québec est grandement facilité par le choix de l'expression "droét d'exister» (de préférence à "patrie») pour traduire «birthdom». Dans l'original ce terme est immédiatement suivi d'une référence à l'Écosse qui, de ce point de vue, se révèle tout aussi gênante et que le traducteur supprime :

[...] new sorrows

Strike heaven on the face, that it resounds

As if it felt with Scotland and yell' $d$ out

Like syllable of dolour. (863. Nous soulignons)

[...] des nouveaux malheurs éclatent

Comme des orages dans l'ciel, si tant qu'tout' not' pau' pays l'ressent

Si tant qu'on peut quasiment l'entendre crier sa douleur.

(116. Nous soulignons)

Non seulement l'Écosse disparaît, mais le ciel reçoit une fonction moins métaphysique, et moins poétique aussi, puisqu'on le ramène prosaïquement à sa réalité météorologique par une désactivation de la symbolique religieuse qui est devenue une anti-valeur dans la société québécoise.

Développant le thème de la nation spoliée, la scène originale présente un très haut degré de compatibilité avec le discours sur la condition québécoise. Cela explique sans doute le nombre particulièrement élevé des transformations axio-idéologiques que la traduction lui fait subir. Ces modifications portent fréquemment sur les déterminants qui tantôt sont neutralisés et tantôt carrément substitués :

[...] I think withal,

There would be hands uplified in my right ; (863. Nous soulignons)

[...] j'pense que ben des bras sont parés à se l'ver

Pour défende nos droéts

(117. Nous soulignons) 
Dans le texte original, l'héritier légitime du royaume d'Écosse jauge l'appui dont il bénéficierait personnellement pour renverser l'usurpateur. Dans la version québécoise, c'est un héros du soulèvement de 1837 que l'on entend, un patriote luttant pour le triomphe de la cause commune : libérer le pays «d'la main damnée qui l'opprime» (1977: 97). Un slogan que n'auraient pas désavoué non plus les militants du Front de libération du Québec durant les événements d'Octobre 1970. N'est-ce d'ailleurs pas sur le fond de l'actualité qui se déroule entre ces événements et le référendum sur la souveraineté que le public est amené à saisir un énoncé comme celui-ci :

Not'cause peut pas ête plus jusse! La victoère nous attend

Au boutte d'la route!

[...]

Bon yeu qu'j'ai hâte qu'les temps changent!

C'tes temps-citte sont en train d'nous virer en étranges !

$(120-121)$

Ces deux séries de propositions sont opportunément rapprochées par la disparition de la «scène de ventilation» et, confrontée à l'original, la traduction de ces mêmes vers manifeste un glissement idéologique très net:

\section{Enter ROSS}

MACDUFF

My ever-gentle cousin, welcome hither.

\section{MALCOLM}

I know him now. Good God, betimes remove

The means that makes us strangers! (864)

Ce passage est univoque: Malcolm ne reconnaît pas immédiatement le général Ross dont il est séparé depuis son exil en Angleterre. Les événements d'Écosse qui les ont tenus éloignés les ont rendus étrangers l'un à l'autre. Garneau donne à «strangers» le sens de «foreigners» dans une tournure répétitive qui laisse au public québécois, menacé d'assimilation et travaillé par le slogan «maîtres chez nous», la faculté de lui assigner ce sens en plus ou à la place du sens contextuel ( $" j$ 'ai hâte qu'les temps changent / C'tes temps-citte sont en train d' nous virer en étranges !»)

La différence entre le texte anglais et la version québécoise est parfois beaucoup plus subtile encore, masquée par le contexte qui paraît l'induire naturellement. Pourtant une écoute plus attentive fait découvrir, en faisceau, de minuscules modifications qui attirent sans ambiguité le texte original dans la sphère du discours social québécois. Écoutons par exemple Macduff réagir lorsqu'il apprend le saccage de ses biens et le massacre de sa femme et de ses enfants:

I cannot but remember such things were,

That were most precious to me. Did heaven look on,

And would not take their part?

(865. Nous soulignons)

C'que j'ava's d'plus précieux dans l'monde, chu t'obligé d'commencer

A m'en souv'nir. Comment c'est que l'bon Dieu peut laisser fère

Des affères pareilles? Sans prende la part des faibes?

(125. Nous soulignons)

La neutralité d'expression de l'objet prédiqué («ce») est conforme à l'expression originale («things»), mais Garneau neutralise ensuite le déterminant personnel («their») 
qu'il transforme en un collectif beaucoup plus englobant ( «les faibes») et d'une disponibilité référentielle que, dans le contexte immédiat de ce qui précède, le spectateur québécois peut combler sans peine, lui que la devise du Québec — «je me souviens» - inscrite jusque sur les plaques d'immatriculation des voitures, enjoint quotidiennement de se rappeler qu'il a perdu, par la conquête britannique, ce que lui-même avait de plus précieux: la liberté, mot clé du discours social québécois. Motivée sans aucun doute par l'original ( «I [...] remember»), la présence en filigrane du slogan «je me souviens» apparaît en toute lumière dans le texte d'arrivée lorsqu'on rapproche la version québécoise de n'importe quelle autre des récentes versions françaises, où le principe de la québécité ne trouverait plus d'ancrage, le faisceau des modifications pertinentes ayant disparu :

Je ne puis pas ne pas me souvenir

Qu'ils existaient, qu'ils furent tout mon bien.

Le Ciel regarde-t-il, sans les défendre?

(Shakespeare 1983: 122)

Ainsi déduira-t-on que l'écart notable entre «I cannot but remember» et «chu t'obligé d' commencer à m'en souv'nir» s'explique par la même nécessité de cohérence discursive, celle que le lieu de la réception impose à la traduction. L'énoncé original exprime un état affectif ( this deadly grief») qu'il faut surmonter ( (Dispute it like a man»). Chez Garneau cela débouche sur une forme d'auto-impératif: je dois désormais me souvenir que j'ai perdu ce que j'avais de plus précieux. Cet impératif à usage individuel est de même nature que l'injonction sociale «je me souviens» adressée sous cette forme personnelle et directe à tous les Québécois. C'est une forme particulière de l'injonction qui, joignant l'acte à la parole, reçoit le nom de performatif dans la théorie des actes de langage. L'efficacité de ce performatif est directement liée à la subjectivation de l'énoncé. Il suffit pour s'en convaincre de comparer «souviens-toi» et «je me souviens». Dans le deuxième cas seulement, dire et faire peuvent se fondre en un même acte de la persuasion, notamment celui de la publicité ( «Moi, je fais ma part!», «J'M», «Moi, j’ embarque!», etc.). On ne s'étonnera donc pas d'en relever la manifestation chez Garneau, là où le texte original ne l'appelle aucunement :

$O$ nation miserable [...]

When shalt thou see thy wholesome days again [...] (864)

$J^{\prime}$ appartiens à eune nation ben miserabe

[...] J'me d'mande si on va

Jama's r'voêr les beaux jours d'avant [...]

(119. Nous soulignons)

Appelée par le registre dans lequel s'expriment les protagonistes de la version québécoise, la subjectivation de l'énoncé amène le texte à coïncider avec le discours de la société réceptrice. Elle est en outre soutenue par l'effacement de la différence entre le plan de la fiction et celui du réel, car la langue de traduction ne correspond pas au code usuel de la représentation québécoise des auvres du grand répertoire. Il s'agit d'un «lecte» ancré distinctement dans la réalité du Québec et d'aucune autre zone de la francophonie, dont l'utilisation inédite produit un effet mobilisateur, voire cathartique, sur l'auditoire québécois. Induite par la langue des dialogues, cette permutation du fictif avec le réel permet au spectateur d'investir le texte de sa propre subjectivité, de son propre vécu individuel et collectif ( J'appartiens à eune nation ben misérabe»). Mais en même temps, la langue rabat le réel sur le symbolique, car pour le spectateur québécois, cette langue est tout aussi marquée que le serait en France une version de Macbeth en 
français régional. Sur la langue standard parlée au Québec, la «langue québécoise» de Michel Garneau se détache par son archaïsme. Elle apparait alors comme une langue édénique, celle du Québec d'avant la conquête britannique, du Québec qui fut libre autrefois. Cette langue que pas un habitué des salles de théâtre ne parle dans la réalité - et Garneau pas plus qu'un autre - acquiert une valeur symbolique qui, par le contexte de la réception, prend force argumentative. Omniprésente et pourtant dissimulée puisque le spectateur, fixé sur les dialogues, l'entend sans l'écouter, elle constitue à elle seule un moyen de persuasion. Elle forme, en d'autres termes, l'assise perlocutoire du message dialogué.

Au Québec, société encore incertaine de son identité, la traduction du théâtre remplit d'abord une fonction spéculaire de nature à légitimer le discours constitutif de la représentation sociale, lui apportant de l'extérieur des appuis qui le confortent. Telle est bien la visée d'une traduction explicitement «québécoise» du monument littéraire appelé Macbeth. Dans cette société où la littérature a partie liée avec le politique et où l'on proclame que l'existence même de la «nation québécoise» est subordonnée à celle d'une littérature propre ${ }^{8}$, le travail de la traduction recoupe celui de l'écriture: consolider les idéologèmes de la représentation sociale au Québec. Cela même implique une mise entre parenthèses de l'altérité fondamentale du texte étranger appelé à cautionner le fait québécois.

Le spectateur qui assiste à une représentation de Macbeth dans la version de Michel Garneau aura l'impression que la tragédie de Shakespeare symbolise admirablement bien la condition québécoise et, en retour, porté par ce grand texte que la traduction a remodelé, le destin du Québec prendra un caractère d'universalité légitimant, au delà des frontières et du temps, le problème québécois érigé en invariant humain.

Le travail perlocutoire de la traduction peut donc être décrit comme cette action sur la conscience du récepteur qui découle de la transformation des présupposés du texte original et de la manipulation du point de vue. Le refaçonnement du texte étranger équivaut en l'espèce à un mode d'argumentation et de persuasion.

Il faudrait parler ici de l'effet stencil de la traduction qui découpe le texte de départ suivant les contours d'une grille que lui impose l'institution discursive du milieu récepteur. Tantôt elle évide le texte et tantôt le remplit. Mais il ne suffit pas d'en conclure, comme on le fait presque toujours, qu'au total le texte d'arrivée dit autre chose que l'original. Par les silences et les gloses qu'il introduit dans le texte, le traducteur manipule la perspective du récepteur sur ce texte; il fixe un point de vue à partir duquel s'organisent une cohérence et une intelligibilité nouvelles. On connaît en peinture le phénomène de l'anamorphose, ce jeu de perspective déformante où la reconstitution de l'image n'est possible qu'à partir d'un seul et unique point de regard. Le lecteur ignorant la langue du texte original est dans une situation comparable à celui qui regarde l'anamorphose d'un endroit qui n'est pas le bon, car l'anamorphose est une déformation codifiée de l'image. Le traducteur, comme le peintre, connaît les règles de la transformation et, comme lui, il construit une représentation intelligible du texte original, c'est-à-dire qu'il fixe aussi le point de vision unique et nécessaire à cette intelligibilité. La déformation est donc relative à ce point de vue, construite ou dissoute par lui. En déterminant le spectateur à voir le texte depuis cette position unique, la traduction s'apparente à une technique d'influence. La traduction de Garneau est ancrée dans un discours sous-tendu par une idéologie de la québécité, une vision du monde québécois. Cette idéologie ouvre et ferme à la fois l'optique dans laquelle le texte shakespearien sera lu. À la manière du collimateur qui rassemble et resserre les rayons divergents, l'idéologie normalise les éléments hétérogènes du texte original - hétérogènes par rapport au discours-cible, qui structure et produit cette idéologie. Par la manifestation du point de vue, le traducteur organise la 
pertinence idéologique du texte étranger dans la société réceptrice. La fonction persuasive de la traduction de Garneau découle de cette régulation puisqu'elle conduit le spectateur à investir de lui-même le texte original refaçonné d'un contenu idéologique conforme à la représentation sociale du Québec. L'ajustement du Macbeth de Shakespeare consiste à renforcer ou à masquer certaines séquences de telle sorte que la représentation de l'Écosse fictive coïncide avec l'entité spatio-temporelle appelée Québec ou plus exactement «Québec libre», car il s'agit d'un lieu-objet autour duquel se cristallise un ensemble d'idéologèmes qui le définissent et le caractérisent en fonction d'une historicité, à travers le discours social contemporain de la traduction.

Dans le schéma qui ouvrait cette analyse apparaissaient trois chronotopes, trois espaces-temps correspondant aux trois plans de lecture possible introduits par la traduction. Ces lectures ne sont pas entièrement coextensives. Un grand nombre de séquences ou de scènes prises individuellement ne se prêtent pas au jeu de la double lecture. Elles n'ont de sens que dans l'espace-temps de la fiction shakespearienne. Mais le travail perlocutoire de la traduction est lié à ces intermittences, car elles endorment la méfiance du récepteur qui jamais ne confondra Macbeth avec une prise de position doctrinale sur le Québec: ses défenses devant Shakespeare sont anesthésiées. La critique, variante du discours social dans ce qu'il a de plus institutionnel, s'y est d'ailleurs laissée prendre9.

Dans la mesure même où elle introduit (y compris par défaut) certaines marques qui déclenchent ou inhibent le comportement de la double lecture et dans la mesure où, en manipulant le poịt de vue, elle amène subrepticement le récepteur à investir l'objet artistique d'un contenu idéologique, la traduction s'apparente à une technique argumentative et transforme le texte esthétique en un texte persuasif. Ainsi, l'analyse de ce cas limite nous fait également voir le rôle du sujet traduisant, qui intériorise les codes discursifs du groupe social dont il se réclame et intervient sur le texte étranger comme un agent de rationalisation. Lieu d'une compétence discursive ${ }^{10}$ comme tout sujet social, le sujet traduisant est donc avant tout un collectif traduisant qui fait de la traduction un des modes de régulation et, par conséquent, d'institutionnalisation du discours venu de l'étranger.

Notes

1. La notion d'acte perlocutoire (ou perlocutionnaire) définie par John Searle (1977 [1969]: 25) concerne ce qu'en traduction on appelle communément l'effet sur le réccpteur: «For example, by arguing I may persuade or convince someone, by warning him I may score or alarm him, by making a request I may get him to do something, by informing him I may convince him (enlighten, edify, inspire him, get him to realize). The italicized expressions above denote perlocutionary acts.»

2. Traduction inédite. Manuscrit non daté déposé à la bibliothèque de l'École nationale de théâtre, Montréal.

3. La notion de discours social désigne tout ce qui se dit ou s'écrit dans une société donnée prise à un moment donné de son histoire. Le discours d'une société se caractérise par des systèmes d'idées (idéologies) liées à des valeurs (axiologie), qui sous-tendent le spectre entier de ses manifestations, quel qu'en soit le champ, depuis le discours de la science jusqu'à celui de la poésie. Nous nous appuyons ici principalement sur les récents travaux de Marc Angenot.

4. Voici comment M. Bakhtine $(1975: 237)$ définit la notion de chronotope: «Nous appellerons chronotope ce qui se traduit, littéralement par «temps-espace»: la corrélation essentielle des rapports spatio-temporels, telle qu'elle a été assimilée par la littérature. Ce terme est propre aux mathématiques; il a été introduit et adapté sur la base de la théorie de la relativité d'Einstein. Mais le sens spécial qu'il a reçu nous importe peu. Nous comptons l'introduire dans l'histoire littéraire presque (mais pas absolument) comme une méta phore. Ce qui compte pour nous, c'est qu'il exprime l'indissolubilité de l'espace et du temps (celui-ci comme quatrième dimension de l'espace). Nous entendrons chronotope comme une catégorie littéraire de la forme et du contenu, sans toucher à son rôle dans d'autres sphères de la culture.

Dans le chronotope de l'art littéraire a lieu la fusion des indices spatiaux et temporels en un tout intelligible et concret. Ici le temps se condense, devient compact, visible pour l'art, tandis que l'espace s'intensifie, s'engouffre dans le mouvement du temps, du sujet, de l'histoire. Les indices du temps se découvrent dans l'espace, celui-ci est perçu et mesuré d'après le temps. Cette intersection des séries et cette fusion des indices spatiaux caractérisent, précisément, le chronotope de l'art littéraire.» 
Ainsi défini, le chronotope est un espace-temps discursif. Pour les besoins de la démonstration, nous avons dissocié les indices spatio-temporels selon qu'ils renvoient exclusivement à l'Écosse fictive de la tragédie shakespearienne ou qu'ils s'appliquent (aussi) à la Nouvelle-France. L'espace-temps (réel et non plus fictif) de la réception enchâsse ces deux chronotopes dramatiques fusionnés dans le texte d'arrivée. Ce dernier vient lui-même s'insérer dans un champ culturel, un espace discursif organisé, qui rend possible l'intégration sémantique de ces trois plans par le récepteur.

5. «Nous appellerons «idéologème» toute maxime, sous-jacente à un énoncé, dont le sujet circonscrit un champ de pertinence particulier (que ce soit «la valeur morale», «le Juif», «la mission de la France» ou «l'instinct maternel»)» (M. Angenot $1977: 24)$. Les «idéologèmes fonctionnent, à l'instar des «lieux» aristotéliciens, comme des principes régulateurs sous-jacents aux discours sociaux auxquels ils confèrent autorité et cohérence [...] les «lieux» de l'ancienne rhétorique ne sont que les plus généraux des «idéologèmes», ceux dont la pertinence historique est plus durable en même temps que le spectre d'application en est le plus large» (1977: 24). «La maxime idéologique est un présupposé du discours («un postulat commun, un lieu commun») [...] Son repérage est indépendant de sa réalisation superficielle. [...] Le présupposé idéologique est commun aux interlocuteurs, quelles que soient leurs divergences ultérieures. [...] Le présupposé est de l'ordre de l'évidence, il est actif mais absent du discours même, parce qu'il ne requiert pas de démonstration. [...] C'est reprendre ici l'hypothèse formulée par Michel Foucault (1969: 94). L'unité du discours «n'est pas dans la cohérence visible et horizontale des éléments formés; elle réside bien en deçà, dans le système qui rend possible et régit une formation».» $(1977: 26-27)$

6. L'institution recouvre ici deux choses: d'une part, «la base matérielle» ou encore «les appareils» (pour l'institution littéraire, par exemple, il s'agira de l'enseignement, de la critique, des éditeurs, du marché ou des prix); d'autre part, les «normes discursives» proprement dites - c'est-à-dire «les règles de production et de lecture» qui, elles, sont implicites (A. Belleau 1981 : 16). Voir également Jacques Dubois (1983), qui fut l'un des premiers à définir les paramètres de l'institution littéraire et son rôle régulateur dans la production et la réception des textes.

7. «On s'accorde aussi à reconnaître deux interpolations importantes, les scènes des sorcières aux III' et IVe actes, qu'on attribue à Thomas Middleton. Moins sûr est-il que les vers 140-155 de la scène 3 de l'acte IV («Le Roi vient-il, s'il vous plât ?», etc.) aient été ajoutés au texte de Shakespeare pour plaire à Jacques $1^{\text {er }}$ : le Folio ayant sans doute été publié à l'occasion de représentations à la Cour» (Bonnefoy 1983:153). Au nom de la pureté du texte, rien ne justifie que le traducteur élimine cette séquence plutôt qu'une autre parmi celles dont parle Bonnefoy, en particulier la scène d'Hécate, personnage surnuméraire, étranger à l'intrigue.

8. Cette affirmation revient comme une obsession chez les écrivains québécois : «La littérature, c'est le signe qu'on existe», déclare par exemple Marcel Dubé, pour qui «Un peuple qui se demande s'il a une littérature, se demande également s'il existe et demeure incapable d'accéder à une réalité extérieure...» Rapporté par A. Parizeau (1986: B2).

9. En voici un exemple parmi d'autres: «La traduction de Macbeth en québécois que nous offre Garneau est fidèle tant au schéma shakespearien qu'à l'écriture tragique. La rupture de ton due à l'usage d'une parlure résolument populaire dont les effets phonétiques sont rendus, aussi bien que les formes lexicales et les tournures syntactiques, n'exclut pas un respect rigoureux des séquences événementielles, des cheminements du discours, des retours mêmes de la pensée [...] toutes les modifications qui vont dans le sens du texte et n'en altèrent que la tonalitế, la traduction est exacte. Elle est l'ouvre d'un poète qui substitue des rythmes familiers de sa langue vernaculaire à ceux que le poète étranger établissait dans son langage [...]» (P. Gobin $1986: 73)$.

10. Nous disons bien «discursive» pour souligner que la compétence linguistique est articulée, au niveau présuppositionnel, sur des idées et des valeurs «coextensives aux pratiques sociales» et dont, par conséquent, dépend «l'intercompréhension des énoncés de surface» dans une société donnée (Angenot 1977). 


\section{BIBLIOGRAPHIE}

ANGENOT, Marc (1977) : «Présupposé, topos, idéologème», Études françaises, vol. 13, nos 1-2, pp. 11-34.

BAKHTINE, Mikhail (1978) : Esthétique et théorie du roman, Paris, Gallimard.

BELLEAU, André (1981) : «Le conflit des codes dans l'institution littéraire québécoise», Liberté, vol. 23, n 2 , pp. $15-20$.

BONNEFOY, Jacques (1983 [1978]) : l'Institution de la littérature, Paris, Nathan

BONNEFOY, Yves (1983): «Notcs», Macbeth, tr. Y. Bonnefoy, Paris, Mercure de France, pp. 153-158.

DUBOIS, Jacques (1983 [1978]) : l'Institution de la littérature, Paris, Nathan.

FOUCAULT, Michel (1969): l'Archéologie du savoir, Paris, Gallimard.

GOBIN, Pierre B. (1986): «Macbête à la foire. De quelques traitements de Shakespeare en français», Études françaises, vol. $19, \mathrm{n}^{\circ} 1$, pp. 67-79.

LEYRIS, Pierre (1977) : «Introduction», Macbeth, tr. P. Leyris, Paris, Aubier-Flammarion, pp. 9-28.

PARIZEAU, Alice (1986) : «Marcel Dubé», la Presse, 8 septembre.

SEARLE, John (1977): Speech Acts, New York, Cambridge University Press.

SHAKESPEARE, William (1977): Macbeth, tr. Michel Garneau, Montréal, VLB.

SHAKESPEARE, William (1983): Macbeth, tr. Yves Bonnefoy, Paris, Mercure de France. 